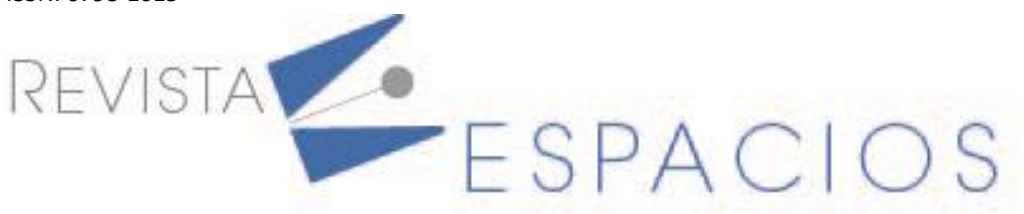

Vol. 41 (43) $2020 \cdot$ Art. 19

\title{
Participación de la mujer indígena en el emprendimiento rural como agente de cambio. Una revisión de literatura
}

\author{
Participation of indigenous women in rural entrepreneurship as an agent of change. A \\ literature review
}

\author{
CASTILLO LÓPEZ, Ana M ${ }^{1}$ \\ ORDOÑEZ ABRIL, Daniel Y.2 \\ GIRALDO VELEZ, Lina C. ${ }^{3}$ \\ GALLEGO MUÑOZ, Dora L. ${ }^{4}$
}

\begin{abstract}
Resumen
La capacidad y acción de las mujeres en diferentes contextos han generado una mayor participación en los escenarios socioculturales e institucionales. El artículo se propone revisar sistemáticamente la literatura sobre la participación de la mujer indígena en emprendimientos rurales como agente de cambio, sintetizando al final los hallazgos. Se encontró relevante la importancia que representa para la mujer indígena tener mayor participación en las diferentes instituciones considerando la necesidad de visibilizar la cultura en estos espacios.

Palabras clave: participación, emprendimiento rural, decolonialidad, mujer indígena.
\end{abstract}

\begin{abstract}
The capacity and action of women in different contexts have generated greater participation in sociocultural and institutional settings. The article aims to systematically review the literature on the participation of indigenous women in rural enterprises as an agent of change, summarizing the findings at the end. In which it was found relevant the importance for indigenous women of having greater participation in different institutions considering the need to make culture visible in these spaces.

key words: participation, rural entrepreneurship, decoloniality, indigenous women.
\end{abstract}

\section{Introducción}

Los estudios sobre la participación de la mujer indígena en el emprendimiento rural han tomado fuerza en los últimos años, principalmente, por la búsqueda del reconocimiento de los derechos a las mujeres, a las minorías étnicas y a la equidad de género. En este panorama las mujeres indígenas llaman particularmente la atención de los investigadores, por enfrentar una situación de doble discriminación por género y etnia Serrano \& Zarza (2013). Es a partir de esta particularidad que el movimiento feminista ha lanzado importantes retos en las últimas dos décadas (Birriel, 2000), donde la institución entendida como cualquier tipo de organización humana con

\footnotetext{
${ }^{1}$ Phd. Research Professor. Faculty of health. LUMEN GENTIUM Catholic University Foundation. amcastillol@unicatolica.edu.co

${ }^{2}$ Research Professor. Faculty of administration, accounting and finance. LUMEN GENTIUM Catholic University Foundation. dyordoneza@unicatolica.edu.co 3 Strategic management specialist. Faculty of administration, accounting and finance. LUMEN GENTIUM Catholic University Foundation. linagiraldo2120@hotmail.com

4 Strategic management specialist. Faculty of administration, accounting and finance. LUMEN GENTIUM Catholic University Foundation. Igallego29@gmail.com
} 
"sistemas de reglas sociales establecidas o inmersas que estructuran las interacciones sociales" (Hodgson, 2011, p.44) juega un rol importante para responder a la resistencia ejercida por las mujeres y sus necesidades.

En ese contexto, la lucha feminista fundamentada en perspectivas teóricas críticas y en el activismo, ha conseguido el reconocimiento de los derechos de las mujeres, como principal mecanismo que ha permitido la participación de las mujeres en los espacios públicos, entre ellos el empleo, la educación, la política, los emprendimientos, los movimientos sociales y la participación con diferentes objetivos. Es decir, la mujer logra estar en procesos de inclusión en el desarrollo como una forma de institucionalizar los principios del feminismo occidental moderno en detrimento de los feminismos subalternos, por su capacidad de actuar en los diferentes entornos poniendo sus habilidades y destrezas en acción (Ahearn, 2016; Belvedresi, 2018; Berbena \& Figueiras, 2014; Pick, Sirkin, Ortega, Xocolotzin \& Giva, 2017; Richardsona, Harperb, Batesa \& Nandib, 2019; Rickard, 2018, y; Araujo, 2017). En cualquier caso esto implica desarrollar un análisis centrado en la decolonialidad del poder y colonialidad del género, a partir de la identificación de la existencia de estructuras de dominación, explotación y exterminio que han sido reproducidas a lo largo de los últimos siglos (Garzón, 2013); Baquero, Caicedo y Rico, (2015).

Por tanto, los estudios de los últimos veinte años se han enfocado en abordar a la mujer indígena y su participación en procesos organizados de tipo económico y la trascendencia de ello en su vida individual, social y económica. Impulsando el crecimiento económico de las regiones rurales, quizás, por el deterioro de la calidad de vida de las familias rurales y la necesidad de sobrevivir (Barron, 1997). Lo que ha permitido que la mujer a través de una serie de resistencias logre ser reconocida como sujeta de derechos en el emprendimiento, tal como lo expone De León (1997), quien refiere que para entender el empoderamiento y el emprendimiento hay que entender la participación de las mujeres en los diferentes escenarios.

Es así que, la mujer emprendedora en los diferentes estudios, no solo es aquella que conforma una empresa o innova en un proceso productivo, sino que además, en el marco del desarrollo local, articula los factores económicos, sociales y políticos e integra a los diferentes sectores y actores de la comunidad, coordina información, necesidades, recursos y busca una fuerte comunicación entre ellos (Formichella, 2002). Aunque es de mencionar que muchos de los estudios muestran que las labores de las mujeres tradicionalmente han estado orientadas a la maternidad, crianza de los niños y cuidado de sus esposos (Kargwell, 2012). Se encuentran estudios como los de Kobeissi (2010) que logran establecer como las brechas salariales y la tasa de fertilidad afecta de manera directamente proporcional los niveles de emprendimiento femenino.

En consecuencia, según Pallarès \& Vera, (2018) el emprendimiento femenino es vital para el desarrollo rural como herramienta con la que se reconoce el esfuerzo de la mujer y su impacto en la sociedad y en la economía local y progreso de su entorno en igualdad de condiciones que el hombre. Esta idea de emprendimiento se basa en las tendencias mundiales, que, según autores como Riaga, Mayorga \& Orozco, (2008); Escobar, (2015), y; Díaz \& Echevarría, (2016), se orienta al crecimiento de la participación de las mujeres en los emprendimientos centrándose en el trabajo y no en el género, reconociéndose la importancia de la mujer para la transformación y productividad de lo rural.

Es decir, las publicaciones más recientes exponen modelos integradores entre el agenciamiento humano, el género, lo étnico y el emprendimiento, donde hay diversos estudios teóricos y empíricos (ver por ejemplo, Ahearn, (2016); Belvedresi, (2018); Devignemont, (2004); Vignemont \& Fourneretb, (2004); Gallagher, (2007); Gamlin, (2020); Lugones, (2010); Medina, (2015); Bravo, (2002); Cañizares \& Fuentes, (2013); Espinosa, (2014); Peredo, (2004); Ruso, (2019); Gómez \& Franco, (2011); Alcaíno, (2013); Mora \& Constanzo, (2017); Zuluaga \& Arango, (2013). 
Por tanto, emerge el siguiente cuestionamiento: ¿cómo caracterizar la literatura sobre la participación de la mujer indígena en los emprendimientos rurales como agente de cambio?. Este articulo presenta de manera panorámica, un repaso de la literatura sobre la participación de la mujer indígena en los emprendimientos rurales como agente de cambio. Para lo que se utilizan como fuentes de datos, publicaciones de revistas indexadas de niveles Q1, Q2, Q3 y Q4, considerando el campo del emprendimiento femenino, rural e indígena. Se adopta como método el repaso de literatura planteado por Cronin, Ryan y Coughlan (2008). De esta manera se pretende proponer posibles focos de investigación que permitan un panorama futuro para el estudio de la mujer indígena y su participación en iniciativas empresariales.

\section{Metodología}

Se usó el protocolo sugerido por Cronin et al. (2008) para alcanar el objetivo de proporcionar una revisión sistémica de literatura que busque identificar, evaluar y resumir todos los estudios pertinentes utilizando un proceso replicable y transparente (Tranfield, Denyer, y Smart, 2003). Este consiste en una metodología basada en una serie de búsquedas en bases de datos, estableciendo prioridades en la selección del material y produciendo al final una síntesis del campo (Creswell, 2010). A continuación, se presenta el paso a paso de la investigación realizada.

\subsection{Selección del tópico de repaso}

El primer paso fue considerar el concepto de la teoría respecto a la mujer indígena. Sustentando con la regla general establecida por Cronin et al. (2008). Por la cual, se establece el recorrido a realizar dentro de la investigación en la medida que se va construyendo con los conceptos de género, emprendimiento femenino, agencia, colonialidad y el papel que juega la mujer indígena.

\subsection{Búsqueda de la literatura}

La búsqueda de literatura fue realizada principalmente en ScienceDirect, Taylor and Francis, Oxford University Press, Springer, Emerald insight y SAGE Journals, Google académico, Scielo, donde se ingresaron diferentes términos de búsqueda y se ajustaron parámetros para acotar los resultados. Cabe señalar que, la elección se dio por ser bases de datos de literatura académica de mayor rigurosidad científica, que indexa una amplia y variada gama de journals de diferentes áreas del conocimiento y cuentan con gran aceptación entre los investigadores.

En las plataformas de datos se adoptaron, en el campo de búsqueda, sólo los descriptores agencia humanas, agency, emprendimiento femenino, feminismo, genero, colonialidad, para definir como objetivo un repaso de la literatura sobre la participación de la mujer indígena en el emprendimiento rural. Las búsquedas fueron filtradas por título y resumen, en un recorte temporal establecido de las últimas tres décadas. Esa opción está conectada a la intención de llenar posibles vacíos existentes desde la aparición del movimiento feminista en los años 60 y 70.

\subsection{Recopilación de literatura, lectura y análisis.}

Las búsquedas realizadas retomaron 100 publicaciones que se encuentran en bases de datos de rigurosidad científica que fueron mencionadas en la sección anterior. A estas se le aplicaron los criterios de exclusión establecidos por Cronin et al., (2008). Quedando seleccionados artículos científicos que han sido debidamente validados (Podsakoff, MacKenzie, Bachrach, y Podsakoff, 2005), por tanto no se limitó la investigación a publicaciones periódicas con el mayor impacto en sus campos, sino que se incluyeron todos los artículos de revistas indexadas por ScienceDirect, Taylor and Francis, Oxford University Press, Springer, Emerald insight y SAGE Journals, Google académico, Scielo, que contenían la expresión "emprendimiento, femenino, rural e 
indígena". Después de esa primera exclusión, la búsqueda arrojo 50 artículos científicos publicados en revistas Q1, Q2, Q3 y Q4, que fueron preliminarmente recolectados.

Posteriormente, se realizó un análisis para verificar si los estudios identificados eran apropiados para los propósitos de esta investigación. Priorizándose artículos que estuvieran escritos en inglés, español y portugués, que contemplaran dentro de sus temas la participación de la mujer indígena en el emprendimiento rural. Siguiendo este procedimiento, se seleccionaron 50 artículos. Una vez definidos los artículos se hizo un análisis individualizado e independiente de estos por parte de los investigadores, tomando como criterios de análisis, el enfoque de investigación; método; muestra cuantitativa y/o cualitativa; técnicas de recolección y análisis de datos; resultados y conclusiones.

En la última parte del estudio, se completó el análisis de contenido y se sistematizaron los 50 artículos, recurriendo al software Nvivo que evidenciarpn interés sobre el tema de la participación mujer indígena en el emprendimiento rural (ver Figura 1). Se extrajo información resumida sobre el tema en términos del tipo de estudio, tipo de análisis, conclusiones, alcance y similitudes entre los estudios. Es decir, se realizó un análisis exploratorio del contenido de los diversos estudios a traves de una representación de las palabras con más de cuatro letras que se mencionaron con mayor frecuencia en las palabras claves de estos artículos, obteniéndose inicialmente 148 palabras que se sintetizaron en la nube de palabras. De estas destacan 58 palabras como emprendimiento femenino, género, indígenas y colonialidad, íntimamente relacionadas con otras palabras de nivel secundario tales como comportamiento de ruralidad, acción, conocimiento, microemprendimientos, modernidad, habilidades, acción, pensamiento práctico y epistemología, lo que se demostrará más adelante en el artículo.

Figura 1

Representación de las 50 palabras (con más de cuatro letras) más utilizadas en los artículos analizados

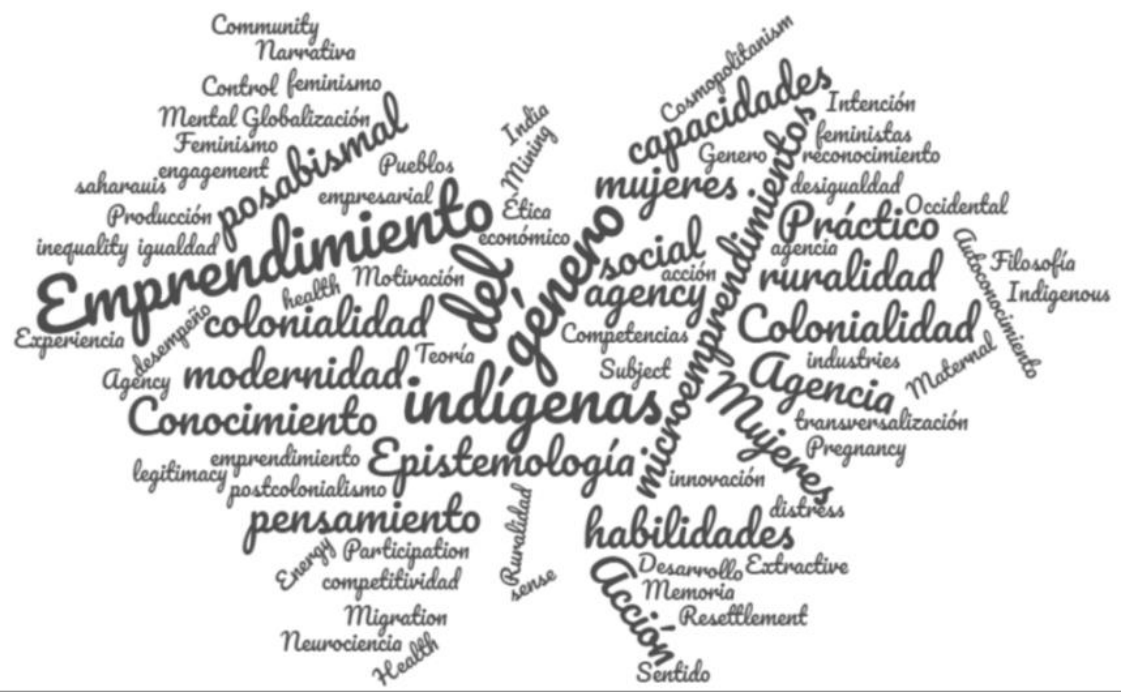

Fuente, propia

\section{Resultados}

\subsection{Colonialidad /decolonialidad}

Para poder realizar un correcto abordaje de la participación de la mujer indígena en el emprendimiento rural se debe partir del paradigma epistemológico occidental que ha supuesto la colonialidad del saber y del ser, haciendo creer que sólo existe una forma posible de conocer el mundo a partir de una racionalidad, una lengua 
y una cultura determinada Esto ha terminado por normalizar la idea de que pensar desde otras categorías cognitivas, lenguas o saberes diferentes es posible si la traducción cultural se acomoda a las pautas dominantes del saber occidental. Por ello, esta perspectiva se inserta dentro de la línea de justificación que concibe a los pueblos indígenas como portadores de un paradigma epistemológico silenciado y negado por la gramática cultural dominante; pero que no pretende ser mejor ni remplazar o superar a las propias del "mundo moderno", entendido según Lander (2000), como la búsqueda de alternativas a la conformación profundamente excluyente y desigual del mundo que exige un esfuerzo de deconstrucción del carácter universal y natural de la sociedad capitalista-liberal. Esto requiere el cuestionamiento de las pretensiones de objetividad y neutralidad de los principales instrumentos de naturalización y legitimación de este orden social; el conjunto de saberes que conocemos globalmente como ciencias sociales. Este trabajo de deconstrucción es un esfuerzo extraordinariamente vigoroso y multifacético que se ha venido produciendo en los últimos años en todas partes del mundo. En este sentido la decolonialidad es la articulación del poder, del saber y del ser, en otras palabras, es salir del esquema de paradigmas donde por su cultura su creencia, multiplicidad de identidades deban seguir las reglas, lineamientos o creencias culturales.

Es bajo este concepto decolonial que las mujeres se han logrado posicionar como productoras, sin embargo, esto tiende a despolitizarse, dado que su trabajo se percibe como una extensión de su rol de cuidadoras, lo que obstaculiza el logro de mayores transformaciones sociales Gómez \& Franco, (2011). Por tanto, se percibe la relación que existe entre colonialidad, desarrollo e igualdad de género, interpretando por colonialidad un modelo de poder cultural enfocado a premisas racistas, etnocentricas y endocentricas; en el que el desarrollo e igualdad de género visualiza a la mujer como objeto pasivo del sistema patriarcal, relación que implica una movilización por los valores, creencias y cosmovisiones determinados por los discursos hegemónicos del podersaber. De ahí que, según Abril \& Cifuentes (2018) el ser humano "se vincule de una forma ambivalente con el poder que le da vida y le somete" (p.45).

\subsection{Agencias humanas}

Las agencias humanas juegan un factor fundamental dentro de la estructura de la sociedad ya que nos permite interactuar, generar y aplicar habilidades, con el objetivo de producir un efecto y obtener cumplimiento de los propósitos independientemente del entorno, cultura, creencias en las que se encuentre ubicado, dicho de otra manera:

La agencia no se refiere a las intenciones que la gente tiene en hacer cosas, pero sí a su capacidad de hacer esas cosas en primer lugar (por eso la agencia implica poder). Agencia se refiere a los eventos de los cuales un individuo es un autor, en el sentido de que un individuo podría, en cualquier fase de una secuencia dada de conducta, haber actuado de manera diferente. (Giddens, 1986, p. 9).

Los orígenes del concepto de agencia, han sido tratados desde Aristóteles quien se enfocó en la reflexión de la acción humana y la libertad de elección implícita en la misma Berbena \& Figueiras, (2014), quien estableció el punto de partida para determinar que la agencia humana es un conjunto de habilidades que permiten actuar intencionalmente con el objetivo de lograr metas, independientemente del contexto donde se encuentre el individuo, guiados por la razón y los sentires que permiten empoderar y ejecutar los propósitos. Siendo así, la temática de la agencia se considera para investigaciones en diversos ámbitos y de diferentes temas. En este trabajo interesa aclarar el modo en que ha sido conceptualizada desde la perspectiva de género, donde se considera un gran aporte para la comprensión de los procesos de configuración de las subjetividades. Para Mahmood (citada por Mora, 2008) la agencia es una "modalidad de acción, que incluye el sentido de sí, las aspiraciones, los proyectos, la capacidad de cada persona para realizar sus intereses, el deseo, las emociones y las experiencias del cuerpo". Es así como de acuerdo a la búsqueda bibliográfica se conceptua la agencia en relación con el género y con el emprendimiento femenino como capacidad y acción y desde una perspectiva 
decolonial como resistencia o resignificación por la represiónes y subordinaciones sufridas por las mujeres, para este caso indígenas.

\subsection{Emprendimiento femenino}

Emprendedor no solo es el que constituye una empresa o innova en un proceso productivo es también alguien que incide en su comunidad ya que coordina y produce condiciones para el desarrollo de los territorios. El emprendimiento femenino, además de lo anterior conlleva de manera transversal la categoría género, es decir un análisis de las particularidades de los emprendimientos masculinos y femeninos, señalando sus diferencias. Para la comprensión de estas diferencias, se toman los aportes de las teóricas feministas (Arango, 2004; Scott, 1996) en cuanto a la relación género-emprendimiento y la relación género-mundo empresarial, relaciones en las que se reproduce la división sexual del trabajo y la hegemonía patrtiarcal en un ejercicio de poder.

Para Hanson (2009), el emprendimiento femenino ha generado la redefinición del rol de la mujer y considera a la perspectiva de género como ineludible para los análisis sobre ello, algunas de las reflexiones que provoca la inclusión de la mirada de género hace referencias a las relaciones con las masculinidades, la emancipación, la reconfiguración de subjetividades femeninas y la extensión de las concepciones de género de acuerdo al contexto y al territorio. En este mismo sentido Castiblanco (2013), conceptúa el emprendimiento femenino de esta menera:

Los estudios sobre emprendimiento femenino a partir de la década de 1990 se han centrado en cuatro elementos fundamentales para su comprensión: las características socio-demográficas de las mujeres que deciden realizar emprendimientos, las diferencias entre emprendimientos de hombres y mujeres, como el contexto puede afectar la decisión de iniciar una actividad productiva y como la definición cultural del género juega un papel preponderante en esa decisión, finalmente el emprendimiento como proceso social es vital en la redefinición del género"p.65.

En países en desarrollo, las mujeres son fundamentales para la sociedad rural como para la economía, aunque se encuentran limitadas, en comparación con los hombres, a los insumos, los servicios, las organizaciones rurales, la infraestructura productiva y la tecnología (FIDA, 2011; 2012). En Latinoamérica, a pesar de visibilizar la contribución de las mujeres al desarrollo rural, y su rol en los ámbitos familiares, sociales, económicos y culturales, la falta de equidad se mantiene como un problema no resuelto (Echevarría y Ribero, 2002; Ruiz y Castro, 2011). Desde una perspectiva generalista, la relación entre empoderamiento femenino y desarrollo rural puede ser analizada desde dos enfoques. Es así que toma sentido cuando Buendía \& Carrasco (2013). Sostienen que:

Desde una perspectiva macro, el empoderamiento femenino va adquiriendo cada vez más relevancia dado su impacto en el desarrollo de los países. En primer lugar, tanto el empoderamiento femenino como el desarrollo rural son realidades complejas. En el primer caso, es necesario un conjunto de intervenciones para promover la participación, aumentar la transparencia, crear capacidades y fortalecer los mecanismos de control en el proceso de desarrollo. En el caso de las mujeres rurales, su capacidad de empoderamiento depende, además, de factores como el acceso a la propiedad sobre la tierra, al empleo y a las actividades generadoras de ingresos, a las infraestructuras, educación, sanidad, servicios financieros y mercados; así como a tener oportunidades para participar en la vida política. (p. 26).

En este sentido el emprendimiento femenino se ve reflejado en el empoderamiento que es movilizado desde el agenciamiento donde se aplica el desarrollo de habilidades, disminuyendo las barreras de acceso a la educación de las mujeres, oportunidades económicas, logrando aumentar la productividad económica; por lo tanto estas emociones contribuyen a generar espacios donde se puedan obtener mejores condiciones de igual de derechos, 
y de esta forma visibilizar el emprendimiento femenino desde todos los contextos sociales y manteniendo el bienestar y las tradiciones socioculturales.

\subsection{Panorama de las Publicaciones}

La relación de las revistas indexadas y sus respectivas clasificaciones se relacionan en la Tabla 1. Se puede notar una concentración de publicaciones en revistas científicas con áreas de estudio enfocadas principalmente, en problemáticas como la mujer y mercado de trabajo; los factores de su desarrollo profesional, estilos de desempeño, emprendimiento femenino, estructura de género de las zonas rurales e igualdad de derechos.

\section{Tabla 1}

Cantidad de publicaciones por revistas indexadas y sus respectivas clasificaciones

\begin{tabular}{|c|c|c|}
\hline Clasificacion & Revista & Cantidad \\
\hline \multirow{11}{*}{ Q1 } & The Extractive Industries and Society & 1 \\
\hline & Annual Review of Social Science \& Medicine & 1 \\
\hline & Consciousness and Cognition & 1 \\
\hline & Energy Research \& Social Science & 1 \\
\hline & Episteme & 2 \\
\hline & Ethical Theory and Moral Practice & 1 \\
\hline & Hypatia & 1 \\
\hline & Organizational Behavior and Human Decision Processes & 1 \\
\hline & Social Science \& Medicine & 2 \\
\hline & WorldDevelopment & 1 \\
\hline & Análisis político & 1 \\
\hline \multirow{5}{*}{ Q2 } & Women'sStudies International Forum & 1 \\
\hline & Economia e Politicalndustriale & 1 \\
\hline & CIRIEC-España, Revista de Economía Pública, Social y Cooperativa & 1 \\
\hline & Revista de economía mundial & 3 \\
\hline & Revista de Estudios Hispánicos & 1 \\
\hline \multirow{9}{*}{ Q3 } & Manuscrito & 1 \\
\hline & Athenea Digital: Revista De Pensamiento E Investigacion Social & 1 \\
\hline & Athenea Digital & 1 \\
\hline & Relaciones Internacionales & 1 \\
\hline & Cuadernos de Administración & 3 \\
\hline & IC - Revista Científica de Información y Comunicación & 1 \\
\hline & Opción & 1 \\
\hline & Revista de Sociología & 1 \\
\hline & Revista Española de Sociología & 1 \\
\hline \multirow{11}{*}{ Q4 } & Interamerican Journal Of Psychology & 1 \\
\hline & Psyche & 1 \\
\hline & Revista De Ciencias Sociales & 1 \\
\hline & El Cotidinano & 1 \\
\hline & Revista La Ventana & 1 \\
\hline & Cepal & 1 \\
\hline & Nera & 1 \\
\hline & Andamios & 3 \\
\hline & Cuadernos de Desarrollo Rural & 4 \\
\hline & Revista.facultad de.ciencias.economia & 1 \\
\hline & Revista de Estudios Empresariales & 4 \\
\hline
\end{tabular}

Fuente: Elaborado a partir de los Cuartiles creados por ScimagoJournal \& CountryRank 2019. 
De igual manera, se evidencia que los artículos clasificados en Q1 tienen un porcentaje de participación del 26\% frente al total de los artículos de investigación, destacándose las revistas Episteme y Social Science \& Medicene con una participación del $8 \%$ del total de artículos consultados. Así mismo se consultaron 5 revistas categorizadas Q2, siendo la Revista de Economía Mundial la de mayor participación con un 6\% del total de revistas analizadas. También se revisaron 11 artículos equivalentes al 22\% del total de revistas analizadas, de los cuales 3 estaban publicados en la revista de Cuadernos de Administración. Finalmente, se hizo la revisión de 19 artículos publicados en revistas categorizadas Q4, encontrándose que la Revista de Estudios Empresariales, la Revista Cuadernos de Desarrollo Rural y la Revista Andamios son las de mayor consulta con un 22 \% del total de artículos revisados.

En cuanto al análisis del dendograma confirma que la participación de la mujer indígena en el emprendimiento rural se ha abordado en diversos campos relacionados con el género, el empoderamiento y el agenciamiento humano, como se refleja en la distancia (Ver Figura 2) entre los artículos analizados, encontrándose 3 grandes conglomerados de artículos que permiten apreciar claramente las relaciones de agrupación entre los datos e incluso entre grupos de ellos, aunque no se muestren las relaciones de similitud o cercanía entre categorías. Observando las sucesivas subdivisiones se puede tener una idea sobre los criterios de agrupación de los mismos y la distancia entre los datos según las relaciones establecidas.

El primer conglomerado aborda artículos que van desde los trabajos de Bravo, (2002) y López, (2004); centrados en la politización radical y en introducir al sujeto como territorio y consecuencia de la propia acción, entendiendo así la noción de agencia como el privilegio de las conexiones y los movimientos frente a las identidades, para explicar la acción. Hasta artículos como los de Díaz, (2014); quien se centra en el subgénero de la escritura conventual, como en el de los estudios de cuestiones indígenas, y funciona para ejemplificar la manera en la que el sujeto colonial se ha convertido en el centro de enfoque en las agendas de investigación.

El segundo conglomerado está compuesto por artículos que van desde los estudios de Matiz, (2009) que buscan realizar una serie de análisis de la investigación académica científica alrededor de la temática del emprendimiento, y llega hasta estudios como los de Jaén, Fernández \& Liñán, (2013); quienes se enfocan en las interacciones entre valores culturales y actividad emprendedora en países con diferentes niveles de ingreso.

Por último, en el tercer conglomerado se encuentran investigaciones que van desde los trabajos de Ahearn (2016); que tratan sobre el estudio del lenguaje, el género, las prácticas de alfabetización y la construcción dialógica de significado y agencia, y van hasta los trabajos de Pacherie (2009); que examinan las posibles relaciones entre diferentes componentes de la experiencia de actuación y los procesos involucrados en la especificación de acciones y el control de acciones en la agencia. Con todo lo anterior es pertinente mencionar la existencia de diferentes posturas frente a cada uno de los temas relacionados con la mujer, emprendimiento, género y colonialidad, convergiendo en la discusión del empoderamiento y papel que desarrollan las mujeres indígenas en los diferentes entornos y situaciones socioculturales que se ven expuestas.

La investigación también consideró los temas estudiados y sus respectivas conclusiones clave (Tabla 2). Resaltando que el mayor número de artículos analizados se enfocó en las agencias humanas relacionadas con la participación de la mujer indígena rural en diferentes iniciativas. Destacándose tres categorías, el agenciamiento, el emprendimiento femenino y la decolonialidad, que buscan poner en evidencia la importancia de la inclusión de la mujer en el área productiva y laboral, hallándose líneas de investigación como: a) estudios sobre el desarrollo social, b) estudios sobre las mujeres indígenas y los derechos humanos, c) estudios sobre el género, la equidad y el desarrollo rural, y d) estudios sobre el emprendimiento femenino en la ruralidad. Investigaciones que en términos generales han encontrado que la incorporación de la mujer al emprendimiento en los últimos años ha sido creciente y constante, al tiempo que sus habilidades son cada vez están más desarrolladas para el logro de sueños por medio del agenciamiento. Y además, donde la colonialidad del poder y la colonialidad del 
género son consecuencia de la dominación y explotación de la mujer, por lo que es vital desarrollar procesos que permitan romper con esas concepciones tradicionales. Por lo tanto se concluye que existe una relación relevante entre las categorías que puntualizan el desempeño del emprendimiento, la visualización y la participación de la mujer indígena en la sociedad y en los diferentes entornos.

\section{Figura 2}

Agrupación de artículos por similitud de palabras Endendograma.

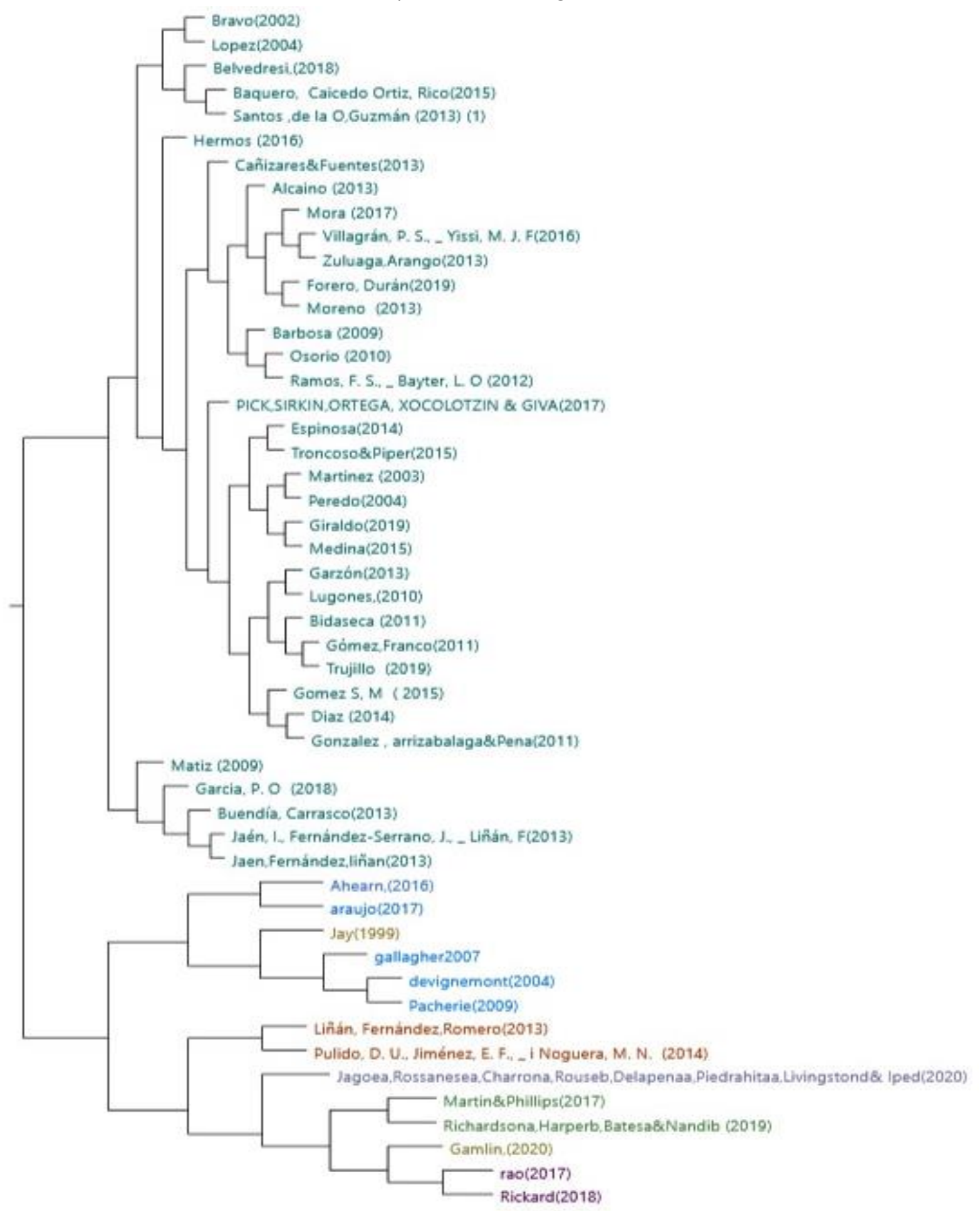

Fuente: Elaboración propia 
Tabla 2

Temas de Análisis

\begin{tabular}{|c|c|c|}
\hline $\begin{array}{l}\text { Tema de } \\
\text { Análisis }\end{array}$ & Principales conclusiones & Estudios \\
\hline $\begin{array}{l}\text { Agencias } \\
\text { Humanas }\end{array}$ & $\begin{array}{l}\text { La agencia femenina tiene una relación mediada socioculturalmente con su capacidad } \\
\text { de actuar. Y su relación con los diferentes entornos. } \\
\text { No hay una precisión en el uso y enfoque del concepto de la definición agencia } \\
\text { humana. } \\
\text { Agencia (racional) en sí, que interactúa causalmente con las creencias normativas del } \\
\text { agente para producir la correspondiente motivación para la acción. } \\
\text { Agencia es la capacidad de establecer vínculos, de articular, de participar junto con } \\
\text { otras. } \\
\text { Sugiere estar abierto a la idea de que algunos pero no todos los procesos dela } \\
\text { producción y el control de la acción dependen de experiencias conscientes y están } \\
\text { listos para reconocer la complejidad de las interacciones cognitivo-experienciales. }\end{array}$ & $\begin{array}{l}\text { Ahearn, (2016); Belvedresi, } \\
\text { (2018); Devignemont (2004); } \\
\text { Vignemont \& Fourneretb, } \\
\text { (2004); Gallagher, (2007); } \\
\text { Berbena \& Figueiras, 2014; } \\
\text { Wallace, (1999); Rao, (2017); } \\
\text { Lopez, (2004); Pacherie, (2009); } \\
\text { Pick, Sirkin, Ortega, Xocolotzin } \\
\text { \& Giva, (2017); Richardsona, } \\
\text { Harperb, Batesa \& Nandib, } \\
\text { (2019); Rickard, (2018); Araujo, } \\
\text { (2017); Díaz, (2014). }\end{array}$ \\
\hline Colonialidad & $\begin{array}{l}\text { Es necesario el análisis de la opresión de género racializada y capitalista, es decir, de } \\
\text { "la colonialidad del género". } \\
\text { Dependiendo del contexto la lucha conjunta implica visibilizar las voces y agencia de } \\
\text { las mujeres. } \\
\text { Pensar la realidad de los países desde la colonialidad del poder y la colonialidad del } \\
\text { género implica entre otras cosas, partir de la existencia de estructuras de dominación, } \\
\text { explotación y exterminio que han sido reproducidas a lo largo de los últimos siglos. } \\
\text { La teoría política en Colombia se encuentra cooptada por la colonialidad del saber, } \\
\text { invitando así a realizar un examen crítico del tipo de ciencia social que quiere } \\
\text { producirse. }\end{array}$ & $\begin{array}{l}\text { Gamlin, (2020), Lugones, } \\
\text { (2010), Medina (2015), } \\
\text { Baquero, Ortiz \& Noguera, } \\
\text { (2015), Bidaseca, (2011), Díaz, } \\
\text { (2014), Garzón (2013), Gómez, } \\
\text { (2015), Trujillo, (2019). }\end{array}$ \\
\hline Ger & $\begin{array}{l}\text { La desigualdad de género se evidencia en la participación de las mujeres en el trabajo } \\
\text { remunerado, así como en la educación, actividades políticas y sociales. } \\
\text { Los proyectos de investigación con una visión de "neutralidad de género", corren el } \\
\text { riesgo de ubicar en una posición de desventaja y marginalización a las mujeres. } \\
\text { los procesos de inclusión de la igualdad de género en el desarrollo son una forma de } \\
\text { institucionalizar subrepticiamente los principios del feminismo occidental moderno en } \\
\text { detrimento de los feminismos subalternos } \\
\text { las mujeres se han posicionado como productoras, pero ello tiende a despolitizarse, } \\
\text { dado que su trabajo se percibe como una extensión de su rol de cuidadoras, lo que } \\
\text { obstaculiza el logro de mayores transformaciones sociales }\end{array}$ & $\begin{array}{l}\text { Martin \& Phillips (2017); } \\
\text { Martinez 2003); Troncoso \& } \\
\text { Piper (2015), .Zuluaga \& } \\
\text { Arango (2013), Alcaíno, (2013), } \\
\text { Gómez \& Franco (2011), Mora } \\
\text { \& Constanzo (2017) }\end{array}$ \\
\hline $\begin{array}{c}\text { Emprendimien } \\
\text { to femenino }\end{array}$ & $\begin{array}{l}\text { Investigadores, entidades de educación, sector privado, gobiernos locales, regionales y } \\
\text { el gobierno nacional, deben trabajar no sólo en la generación de una cultura que } \\
\text { privilegie el emprendimiento como opción de vida y herramienta de desarrollo. } \\
\text { La globalización de la economía está definiendo nuevos escenarios para el desarrollo } \\
\text { de las actividades locales, y esto altera de manera notable las relaciones económicas } \\
\text { tradicionales, mediante un empuje hacia la liberalización de los mercados por su } \\
\text { contribución a la actividad económica, y a la capacidad de innovar y evolucionar de los } \\
\text { espacios. } \\
\text { El negocio ha cambiado la percepción del trabajo de las mujeres rurales y las ha } \\
\text { cambiado a ellas mismas, reafirmando de este modo que ser emprendedoras no es } \\
\text { solo una actividad económica, sino también, como ha destacado muy bien Bröckling } \\
\text { (2015), una forma de concebirse a sí mismo y a los otros. } \\
\text { Las mujeres han sido capaces de tomar decisiones a nivel del negocio, dado que ser } \\
\text { emprendedor(a) entraña un riesgo económico, físico y personal y una habilidad de } \\
\text { movilizar y gestionar recursos en pos de una ganancia, una ocupación y una } \\
\text { satisfacción personal. } \\
\text { Alcanzar los nuevos estándares de calidad, de mercados crecientemente exigentes, } \\
\text { aparece como un desafío en la gestión de las microempresas rurales femeninas en el } \\
\text { marco de la nueva ruralidad. } \\
\text { la desigualdad de género tiene una relación mayor con el emprendimiento por } \\
\text { necesidad, tanto en hombres como en mujeres. } \\
\text { Los emprendedores son iniciadores de cambio y sobre todo generadores de nuevas } \\
\text { oportunidades, busca continuamente crear riqueza. }\end{array}$ & $\begin{array}{l}\text { Barbosa, \& Sandoval. (2011), } \\
\text { Buendía \& Carrasco, (2013), } \\
\text { Forero \& Durán (2019), García, } \\
\text { \& Capitán, (2018), Hermoso. } \\
\text { (2016), Jaén, Fernández \& } \\
\text { Liñán, (2013), Liñán., } \\
\text { Fernández \& Romero (2013), } \\
\text { Matiz (2009), Moreno, (2013), } \\
\text { Osorio, Gálvez \& Murillo } \\
\text { (2010), Pulido., Jiménez, \& i } \\
\text { Noguera, (2014), Ramos, \& } \\
\text { Bayter, (2012), Santos, Barroso, } \\
\text { \& Guzmán. (2013), Villagrán, \& } \\
\text { Yissi, (2016)), Bravo (2002), } \\
\text { Cañizares \& Fuentes (2013), } \\
\text { Espinosa (2014), Peredo } \\
\text { (2004), Ruso (2019). }\end{array}$ \\
\hline
\end{tabular}

Fuente, elaboración propia 


\subsection{Tipos de estudios}

Finalmente, se analizó la tipología de los estudios con respecto al tipo de análisis realizado. Como se establece en la Tabla 3, en la que se muestran 23 estudios puramente teóricos, y 27 son empíricos, 10 son estudios cuantitativos donde la mayoría emplean técnicas estadísticas y econométricas como el análisis de ecuaciones estructurales y aplicando las estadísticas descriptivas, y 17 artículos son cualitativos donde más del $47 \%$ se enmarcan en análisis del discurso. Por lo tanto, el análisis de la Tabla 3. Confirma que las metodologías cualitativas son las más desarrolladas en este tipo de investigaciones. Sin embargo, es necesario realizar investigaciones mixtas que contribuyan a llenar los vacíos teóricos al tiempo que se logra validar econométricamente los mismos.

Tabla 3

Tipo de Estudio y Tipo de Análisis

\begin{tabular}{|c|c|c|c|}
\hline \multicolumn{2}{|c|}{ Tipo de Estudio } & Tipo de Análisis & Referencias \\
\hline \multicolumn{2}{|l|}{$\begin{array}{l}\text { Revisión } \\
\text { teórica }\end{array}$} & $\begin{array}{l}\text { Revisión de literatura } \\
\text { sistemática }\end{array}$ & $\begin{array}{l}\text { Ahearn, (2016); Belvedresi, (2018); Vignemont \& Fourneretb, (2004); } \\
\text { Gallagher, (2007); Berbena \& Figueiras, (2014; Wallace, (1999); López, } \\
\text { (2004); Lugones, (2010); Medina, (2015); Bravo, (2002); Espinosa, (2014); } \\
\text { Ramos, \& Bayter, (2012); Osorio, Galvéz \& Murillo (2010); Martínez, } \\
\text { (2003); Troncoso \& Piper, (2015); Santos, Barroso y Guzmán, (2013); Matiz, } \\
\text { (2009); Alcaíno, (2013); Forero \& Durán (2019), Díaz, (2014); Garzón } \\
\text { (2013); Moreno, (2013); Gómez \& Franco (2011), }\end{array}$ \\
\hline \multirow{12}{*}{$\begin{array}{l}\text { Estudios } \\
\text { Empíricos }\end{array}$} & \multirow[t]{6}{*}{ Cuantitativo } & Regresión Lineal & Jaén, Fernández \& Liñán, (2013); \\
\hline & & Estadísticas descriptivas & $\begin{array}{l}\text { Martin \& Phillips, (2017); García \& Capitán, (2018); Pick,Sirkin,Ortega, } \\
\text { Xocolotzin \& Giva(2017), Hermoso. (2016). }\end{array}$ \\
\hline & & GHQ12 & Richardsona, Harperb, Batesa \& Nandib, (2019) \\
\hline & & $\begin{array}{l}\text { Análisis Univariante y } \\
\text { Bivariante }\end{array}$ & Cañizares \& Fuentes, (2013) \\
\hline & & Ecuaciones estructurales & $\begin{array}{l}\text { Barbosa \& Sandoval, (2011); Liñán, Fernández \& Romero, (2013); Buendía } \\
\text { \& Carrasco, (2013); }\end{array}$ \\
\hline & & Regresión logística & Pulido, Jiménez \& Noguera, (2014); \\
\hline & \multirow[t]{6}{*}{ Cualitativo } & Análisis del discurso & $\begin{array}{l}\text { Aaraujo (2017), Ruso (2019), Trujillo (2019), Mora \& Constanzo (2017); } \\
\text { Bidaseca, (2011); Villagrán, \& Yissi, (2016); Devignemont (2004), Rickard } \\
\text { (2018) }\end{array}$ \\
\hline & & Fenomenológico & Pacherie (2009) \\
\hline & & Análisis de Reastabilidad & Rao (2017) \\
\hline & & Análisis de contenido & Gómez, (2015); Baquero, Caicedo \& Rico (2015) \\
\hline & & $\begin{array}{l}\text { Análisis temático } \\
\text { inductivo }\end{array}$ & Gamlin, (2020), Peredo (2004) \\
\hline & & Análisisetnográfico & Zuluaga, \& Arango (2013); Díaz (2014) \\
\hline
\end{tabular}

\section{Implicaciones, futuras líneas de investigación y conclusiones}

Sobre la base de los resultados presentados por los diferentes autores, se puede identificar unas similitudes en el abordaje de los distintos temas que evidencian la importancia de la participación de la mujer rural y en particular la indígena en los diferentes territorios y comunidades. Se identificaron las siguientes categorías: emprendimiento femenino, decolonialidad, género y agencia humana, que fueron analizadas de acuerdo a la revisión de la literatura para responder al objetivo principal de este artículo.

En este sentido el perfil emprendedor desde la perspectiva de Gartner (1989), se encuentra compuesto por las características personales, sociales y psicologías, ubicando dentro de cada una de ellas un conjunto de competencias dentro de las cuales se encuentra la autonomía, la toma de decisiones, la responsabilidad, el liderazgo, trabajo en equipo, la necesidad de logro, entre otras. Ahora bien, se debe entender por competencia lo planteado por McClelland (citado por Ortiz et al., 2013) como algo que realmente causa un rendimiento 
superior en el trabajo, anteponiendo su significado y dimensión al enfoque educativo, para ponerlo en correspondencia con otros elementos alternativos como el género, etnia o clase social para medir el rendimiento laboral de la persona en el contexto organizativo.

Por ende, se hace necesario proponer futuras líneas de investigación, que busquen profundizar más sobre el tema de mujeres indígenas en los diferentes sectores y contextos, llevando este conocimiento a la sociedad con el fin de ampliar en entendimiento de sus costumbres y creencias llevadas dentro de los diferentes roles. Por lo tanto la pregunta que se queda por resolver seria ¿Cómo interactúa la agencia con las creencias normativas y hegemónicas de género de las mujeres indígenas para motivar la acción en los diferentes roles asignados por la sociedad?

\subsection{Conclusiones}

En el histórico las mujeres rurales, vienen transformando o reconstruyendo una visión de los paradigmas o esquemas impuestos, siendo generadoras de cambio respecto a la desigualdad de género que se ve marcada por las tradiciones culturales. Por tanto, se puede considerar que la participación de la mujer rural y en particular la indígena ha permitido incursionar en proyectos sociales de emprendimiento en el sector rural generando en ellas una transformación y empoderamiento, sin perder sus costumbres y tradiciones; Pero adquiriendo capacidades emprendedoras que le permiten aprovechar las oportunidades de desarrollo sostenible en el tiempo.

Ahora bien, después de realizar el recorrido por la literatura revisada, se puede concluir que hablar de emprendimiento femenino, es hablar de una relación marcada con el empoderamiento y agenciamiento donde las mujeres por medio de sus habilidades, y emociones, logran participar activamente en diferentes escenarios, generando nuevas oportunidades de desarrollo sostenible en el tiempo. Evidenciando que para movilizar el emprendimiento femenino de la mujer rural y en particular la indígena, se deben articular y profundizar en temas como el agenciamiento, el género, y la decolonialidad, dado que el trabajo de la mujer requiere tener una mayor visibilizacion, que le permita establecer vínculos emocionales movilizados desde el agenciamiento para producir una motivación y lograr un impacto con mayor participación en la sociedad.

\section{Referencias bibliográficas}

Abril, D. Y. O., \& Cifuentes, C. I. A. (2018). De la relación del manager y el empleado a la aproximación del deseo del individuo. Revista Universitária Ruta, 20(1), 33-48.

Ahearn, L. (2016). Narrative paths of native american resistance: tracing agency and commemoration in journalism texts in Eastern North Carolina, 1872-1988.

Alcaíno, L. I. (2013). Problemática de las mujeres en el mercado de trabajo, el espíritu emprendedor en nuevos negocios y relación con sus valores y creencias. Revista de Estudios Empresariales. Segunda Epoca, (1).

Araujo, N. (2018, March). Engendering cosmopolitanism: Gendered narratives of instability and agency. In Women's Studies International Forum (Vol. 67, pp. 102-109). Pergamon.

Baquero, S., Ortiz, J. \& Noguera, J. (2015). Colonialidad del saber y ciencias sociales: una metodología para aprehender los imaginarios colonizados. Análisis político, 28(85), 76-92.

Barbosa, D. M. E., \& Sandoval, A. A. (2011). Capacidades y recursos que influyen en las empresas femeninas. Cuadernos de Administración, 27(45), 31-44.

Belvedresi, R. E. (2018). Historia de las mujeres y agencia femenina. Epistemología e Historia de la Ciencia, 3. 
Berbena, M. A. Z., \& Figueiras, S. C. (2014). Fenomenología de agencia y educación. Notas para el análisis del concepto de agencia humana y sus proyecciones en el ámbito educativo. Magister, 26(2), 98-104.

Bidaseca, K. (2011). "Mujeres blancas buscando salvar a mujeres color café": desigualdad, colonialismo jurídico y feminismo postcolonial. Andamios, 8(17), 61-89.

Bravo, F. (2000). La naturaleza de la acción según Aristóteles. Episteme, NS, 20(2), 17-89.

Buendía, I., \& Carrasco, I. (2013). Mujer, actividad emprendedora y desarrollo rural en América Latina y el Caribe. Cuadernos de desarrollo rural, 10(72), 21-45.

Cañizares, S. M. S., \& García, F. J. F. (2013). Mujer y emprendimiento: Un análisis en el contexto universitario español. Revista de Ciencias Sociales (Ve), 19(1), 140-153.

Castiblanco, S. (2013). Participación de la mujer indígena, en el emprendimiento rural como agente de cambio.Rev.fac.cienc.económicas Vol XXI (2). P.53-66

Creswell, J. (2010). Mapping the developing landscape of mixed methods research. SAGE handbook of mixed methods in social \& behavioral research, 2, 45-68.

Cronin, P., Ryan, F., \& Coughlan, M. (2008). Undertaking a literature review: a step-by-step approach. British jornal of nursing, 17(1), 38-43.

De Leon, A. (1997). Resistencia y utopía: memorial de agravios y crónica de revueltas y profecías acaecidas en la provincia de Chiapas durante los últimos quinientos años de su historia. Ediciones Era.

De Vignemont, F. (2004). The marginal body. In Gurwitsch's Relevancy for Cognitive Science (pp. 131-149). Springer, Dordrecht.

De Vignemont, F., \& Fourneret, P. (2004). The sense of agency: A philosophical and empirical review of the "Who" system. Consciousness and Cognition, 13(1), 1-19.

Díaz-Fernández, I., \& Echevarría-León, D. (2016). El emprendimiento en Cuba: un análisis de la participación de la mujer. Entramado, 12(2), 54-67.

Díaz, M. (2014). El" nuevo paradigma" de los estudios coloniales latinoamericanos: un cuarto de siglo después. Revista de Estudios Hispánicos, 48(3), 519-547.

Forero, L., \& Durán, L. (2019). Aportes a la construcción del estado de arte del emprendimiento femenino en Colombia. Revista Escuela de Administración de Negocios, (86).

Echevarría, P., y Ribero, M. (2002). Nueva ruralidad. Visión del territorio en América Latina y el Caribe. San José: Instituto Interamericano de Cooperación para la Agricultura (IICA).

Ema López, J. E. (2004). Del sujeto a la agencia (a través de lo político). Athenea Digital: Revista de pensamiento e investigación social, (5), 1-24.

Escobar, A. (2015). Territorios de diferencia: la ontología política de los "derechos al territorio". Cuadernos de antropología social, (41), 25-38.

Espinosa-Miñoso, Y. (2014). Una crítica descolonial a la epistemología feminista crítica. El cotidiano, (184), 7-12.

Formichella, M. M. (2002). Educación y Pobreza: Una explicación de los círculos viciosos existentes entre ambas (Doctoral dissertation, Tesis de Grado, Departamento de Economía, Universidad Nacional del Sur). 
Gallagher, S. (2007). The natural philosophy of agency. Philosophy Compass, 2(2), 347-357.

Gamlin, J. B. (2020). "You see, we women, we can't talk, we can't have an opinion...". The coloniality of gender and childbirth practices in Wixárika indigenous families. Social Science \& Medicine, 112912.

García, P. O., \& Capitán, Á. O. (2018). Factores competenciales en el emprendimiento de la mujer. RES. Revista Española de Sociologia, 27(3), 413-432.

Gartner, W. B. (1989). Some suggestions for research on entrepreneurial traits and characteristics. Entrepreneurship theory and practice, 14(1), 27-38.

Garzón López, P. (2013). Pueblos indígenas y decolonialidad: sobre la colonización epistemológica occidental. Andamios, 10(22), 305-331.

Giddens, A. (1986). The Constitution ofSociety: Outline of the Theory ofStructuration (Cambridge: Polity).

Gómez-Quintero, J. D., \& Franco Martínez, J. A. (2011). La agenda oculta de la igualdad de género en el desarrollo. Andamios, 8(17), 37-60.

Gómez, S. M. (2015). Feminidades racializadas e imaginarios coloniales en el humor gráfico de Cuba en el s. XIX. IC Revista Científica de Información y Comunicación, (12).

Hanson, S. (2009) Changing places through women's entrepreneurship Economic Geography. 85 (3)

Hermoso, Ú. (2016). Análisis del emprendimiento femenino atendiendo a la influencia del rol de la mujer en el acceso al mercado laboral ya la educación superior. Opción, 32(10), 394-411.

Hodgson, G. M. (2011). ¿ Qué son las instituciones?. Revista CS, 17-53.

Jaén, I., Fernández, J., \& Liñán, F. (2013). Valores culturales, nivel de ingresos y actividad emprendedora. Revista de economía mundial, (35), 35-51.

Kargwell, S. A. (2012). A comparative study on gender and entrepreneurship development: still a male's world within UAE cultural context. International Journal of Business and Social Science, 3(6).

Kobeissi, N. (2010). Gender factors and female entrepreneurship: International evidence and policy implications. Journal of international entrepreneurship, 8(1), 1-35.

Lander, E. (2000). Ciencias sociales: saberes coloniales y eurocéntricos (pp. 11-40). Buenos Aires: Clacso.

Liñán, F., Fernández, J., \& Romero, I. (2013). Emprendimiento por necesidad y por oportunidad: El efecto mediador de la cultura. Revista de Economía Mundial, (33), 21-47.

Lugones, M. (2011). Hacia un feminismo descolonial. Revista La manzana de la discordia, 6(2), 105-117.

Martin, A. E., \& Phillips, K. W. (2017). What "blindness" to gender differences helps women see and do: Implications for confidence, agency, and action in male-dominated environments. Organizational Behavior and Human Decision Processes, 142, 28-44.

Matiz, F. J. (2009). Investigación en emprendimiento, un reto para la construcción de conocimiento. Revista Escuela de Administración de Negocios, (66), 169-182.

Medina Martin, R. (2014). Mujeres Saharauis, Colonialidad del Género y Nacionalismos: un acercamiento a partir de los feminismos decoloniales. Relaciones Internacionales. 
Mora, A. (2008). Cuerpo, género, agencia y subjetividad. V Jornadas de Sociología de la UNLP, 10, 11 y 12 de diciembre de 2008, La Plata, Argentina.

Mora, G. \& Constanzo, J. (2017). 'Emprender sin descuidar la casa': posiciones y dinámicas organizativas en una asociación productiva de mujeres rurales. Cuadernos de Desarrollo Rural, 14, 90-107.

Moreno, S. E. C. (2013). La construcción de la categoría de emprendimiento femenino. Revista Facultad de Ciencias Económicas, 21(2), 53-66.

Osorio, Gálvez, \& Murillo, (2010). La estrategia y el emprendedor: diversas perspectivas para el análisis. Cuadernos de Administración (Universidad del Valle), (43), 65-80.

Ortiz, V., Prowesk, K., Rodríguez, A., Lesmes, C., \& Ortiz, F., (2011). Definición y clasificación teórica de las competencias académicas, profesionales y laborales. Las competencias del psicólogo en Colombia. Psicología desde el Caribe, (28), 133-165.

Pacherie, E. (2007). The sense of control and the sense of agency. Psyche, 13(1), 1-30.

Pallarès, M., \& Vera, A. (2018). Emprendimiento e innovación de las mujeres: hacia una mayor sostenibilidad en las áreas rurales de montaña. Cuadernos geográficos de la Universidad de Granada, 57(3), 36-57.

Peredo Beltrán, E. (2004). Una aproximación a la problemática de género y etnicidad en América Latina. CEPAL.

Pick, S., Sirkin, J., Ortega, I., Osorio, P., Martínez, R., Xocolotzin, U., \& Givaudan, M. (2007). Escala para medir agencia personal y empoderamiento (ESAGE). Interamerican Journal of Psychology, 41(3), 295-304.

Podsakoff, MacKenzie, Bachrach, \& Podsakoff, (2005). The influence of management journals in the 1980s and 1990s. Strategic Management Journal, 26(5), 473-488.)

Pulido, Jiménez, \& Noguera, (2014). Emprendimiento social femenino y contexto socio-cultural: un análisis internacional. Revista de Estudios Empresariales. Segunda Época, (2).

Ramos, F. S., \& Bayter, L. O. (2012). Emprendimiento y Economía Social, oportunidades y efectos en una sociedad en transformación. CIRIEC-España, revista de economía pública, social y cooperativa, (75), 128151.

Rao, N. (2017). Assets, agency and legitimacy: towards a relational understanding of gender equality policy and practice. World development, 95, 43-54.

Rickard, S. (2019). Gender, agency and decision making in community engagement: Reflections from Afghanistan's Mes Aynak Mine. The Extractive Industries and Society.

Richardson, R., Harper, S., Bates, L. \& Nandi, A. (2019). The effect of agency on women's mental distress: A prospective cohort study from rural Rajasthan, India. Social Science \& Medicine, 233, 47-56.

Riaga, C., Mayorga, D. \& Orozco, Y. (2008). Una revisión a la investigación en emprendimeinto femenino. Revista Facultad de Ciencias Económicas: investigación y reflexión, 16(1), 85-104.

Ruiz, P. y Castro, M. (2011). La situación de las mujeres rurales en América Latina. En J. Anderson et al., Mujer rural: cambios y persistencias en América Latina (pp. 1-36). Lima: Cepes

Santos, F. J., Barroso, M. D. L. O., \& Guzmán, C. (2013). La economía global y los emprendimientos sociales. Revista de economía mundial, (35), 177-196. 
Serrano, H., \& Zarza, M. (2013). Roles sociales mestizos e indígenas: efectos en violencia derivada de rituales y tradiciones. Ra Ximhai, 9(3), 81-97.

Troncoso Pérez, L. E., \& Piper Shafir, I. (2015). Género y memoria: articulaciones críticas y feministas. Athenea digital: revista de pensamiento e investigación social, 15(1), 0065-90.

Villagrán, P. S., \& Yissi, M. J. F. (2016). Ser mujer microempresaria en el medio rural. Espacios, escalas y redes. Cuadernos de Desarrollo Rural, 13(77), 141-165.

Wallace, R. J. (1999). Three conceptions of rational agency. Ethical Theory and Moral Practice, 2(3), 217-242.

Zuluaga, G., \& Arango, C. (2013). Mujeres campesinas: resistencia, organización y agroecología en medio del conflicto armado. Cuadernos de Desarrollo Rural, 10(72), 159-180.

\section{Esta obra está bajo una Licencia Creative Commons Attribución-NoCommercial 4.0 International \\ (cc) BY-NC}

